Textures and Microstructures, 1987, Vol. 7, pp. 1-10

Photocopying permitted by license only

(C) Gordon and Breach Science Publishers Inc., 1987

Printed in the United Kingdom

\title{
Anisotropy of Microstructure and Strength in Fiber Textured Molybdenum Alloys
}

\author{
T. OYAMA $\dagger$ and J. WADSWORTH $\ddagger$
}

$\dagger$ Department of Materials Sciences and Engineering, Stanford University, Stanford, CA 94305. $¥$ Metallurgy Department, Lockheed Palo Alto Research Laboratory 0/93-10, B/204, 3251 Hanover Street, Palo Alto, CA 94304.

(Received 9 June 1986)

Molybdenum and molybdenum alloys exhibit brittle behavior in the transverse direction of wrought bar stock despite having good ductility in the longitudinal direction. This is believed to be due to the presence of cracked-carbide stringers on adversely oriented grain boundaries. In the present paper, the possible role of anisotropy in strength, as a result of the presence of a strong fiber texture, is investigated. It is concluded, both theoretically and experimentally, that anistropy in strength between the transverse and longitudinal direction of barstock containing a perfect fibre texture is not a factor promoting brittle behavior.

\section{INTRODUCTION}

Investigations have recently been carried out to determine the cause of the extremely poor transverse ductility that is found in commercial, wrought, molybdenum bar stock (Wadsworth et al., 1984a, 1984b). The major conclusions from these studies have attributed this particular brittle behavior phenomenon to the presence of cracked carbide stringers on unfavorably oriented grain boundaries. It has also been shown that by careful recrystallization and forging procedures, small molybdenum components can be manufactured that have reasonably isotropic properties (Wadsworth et al., 1984b). 
One of the interesting metallurgical features of bar stock of BCC metals, such as molybdenum, is the development of pronounced [110] fiber textures. These textures are associated with manufacturing methods typically used in the production of bar stock such as extrusion, rotary swaging, and round rolling. It has been recognized for many years that the presence of a [110] fiber texture can lead to wide, curved, ribbon-like grains during constrained deformation due to the orientation of the principal slip systems (Hosforth, 1964; Peck and Thomas, 1961). As described by Hosforth (1964), the development of wide, curved grains can be readily understood from the orientation of the BCC unit cell in the [110] fiber texture under uniaxial tensile stress. In this case, anisotropy of slip leads to favored deformation on limited systems. Thus, an initially spherical grain, instead of becoming cigar-shaped upon subsequent deformation, develops an elliptical cross section. In a polycrystalline aggregate, the various rotational misorientations amongst adjacent grains led to constraints in the free development of these elliptical cross sections. As a result of these contiguity requirements, the grains become wide and curved about the [110].

One of the questions that does not appear to have been uniquely addressed in all the above studies is the role that the fiber texture has on brittle behavior in the transverse orientation of wrought bar stock. It is known, however, that increases in yield strength can promote brittle behavior in BCC metals by making cleavage or grain boundary fracture more easy than general plastic yield (Tietz and Perkins, 1964).

\section{EXPERIMENTAL DETAILS}

Commercial barstock of both wrought TZM (Titanium-Zirconium Molybdenum) and unalloyed (low carbon) molybdenum were used in the present study. The alloys were commercial grade barstock that met the required purity levels (Wadsworth et al., 1984a). Small right-circular cylindrical samples $(8 \mathrm{~mm}$ length $\times 5 \mathrm{~mm}$ diam.) were machined from the bars. Longitudinal samples were machined such that the gage length was parallel to the working direction. Transverse samples were machined such that the gage lengths were either radially or circumferentially oriented with respect to the 
working direction. Compression tests were carried out in an Instron testing machine at a crosshead rate of $0.50 \mathrm{~mm} / \mathrm{min}$.

\section{RESULTS AND DISCUSSION}

As a result of the studies described in the introduction (Wadsworth et al., 1984a, 1984b), it was concluded that poor ductility in the transverse direction of wrought Mo and TZM is a result of cracks associated with carbides on grain boundaries. These grain boundaries are, in addition, unfavorably oriented and large in the transverse direction because of their morphology which, in turn, is influenced by a [110] fiber texture. Examples of such curved grains in molybdenum are shown macroscopically in Figure 1. It should be noted (as originally pointed out by Peck and Thomas (1961)) that this microstructural anisotropy is not apparent in the longitudinal sections of such materials. As shown in Figure 2, longitudinal sections do have the nominal appearance; however, it is quite possible that a single grain is represented several times or more in such an orientation as a result of the curved nature of the grains in the transverse section. An example of the transverse section at high magnification is also shown in Figure 2.

The development of grain boundary cracks, as shown in Figure 3, and the ease of grain boundary separation in general, may be aided by intergranular stresses arising from the presence of a [110] fiber

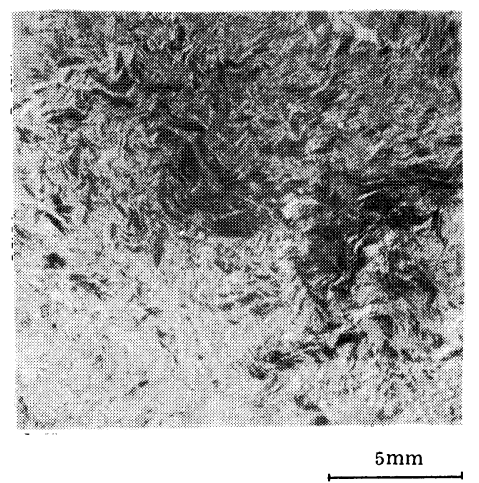

FIGURE 1 Transverse cross-section from the center of unalloyed molybdenum bar stock (37 mm diam.) showing curved grains. 


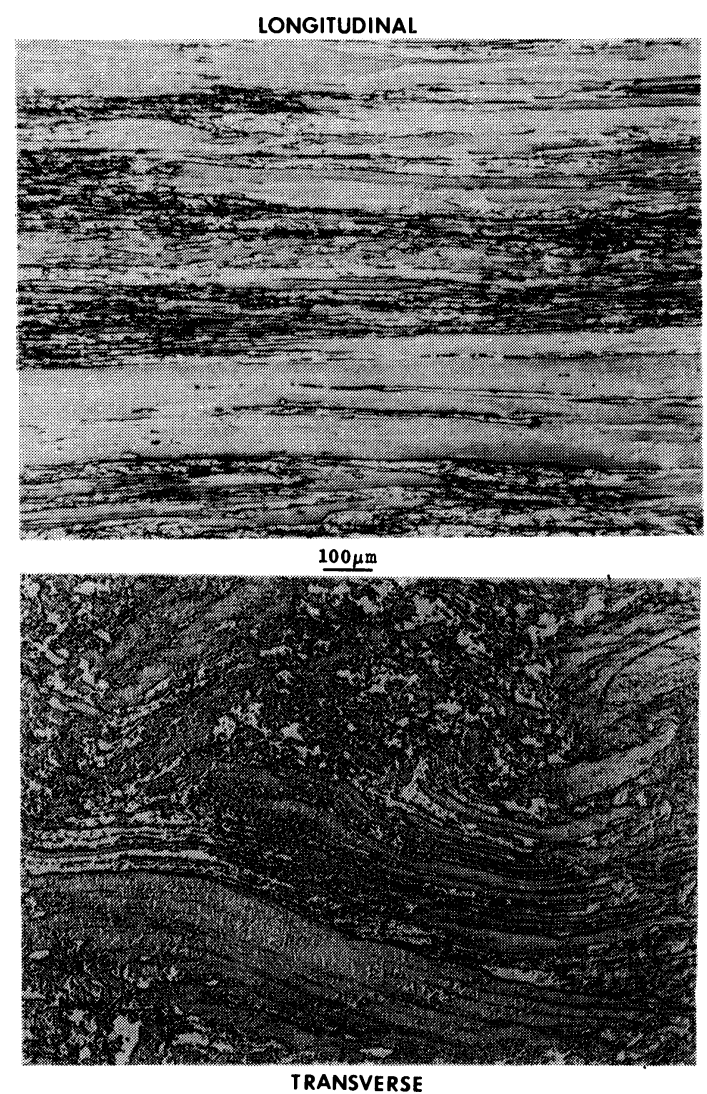

FIGURE 2 Longitudinal and transverse sections of unalloyed molybdenum bar stock.

texture. It should be pointed out that oxygen contamination at grain boundaries has been eliminated as a source of embrittlement in the case of poor transverse ductility in molybdenum barstock (Wadsworth et al., 1984a).

Because there is a pronounced difference between the fracture behaviour in the longitudinal and transverse directions of Mo bar stock, the question of anisotropy in strength is also raised, i.e., is there a difference between the yield strengths in the transverse and longitudinal directions in a bar containing a perfect [110] fiber texture? And, if so, could this be a factor contributing to brittle 


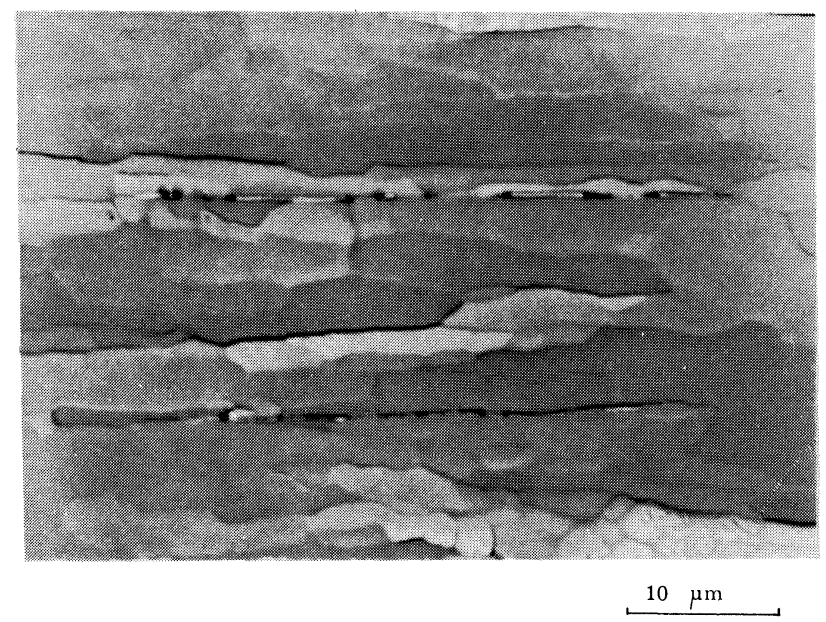

FIGURE 3 High magnification optical photomicrograph of a longitudinal section of wrought, unalloyed molybdenum bar stock. The grain boundaries contain crackedcarbide stringers.

behaviour? A theoretical band experimental study was performed to determine the answer to this question.

The orientation of a unit cell in an extruded bar is shown in Figure 4. It is probable that the [110], [321], and [211] slip planes all operate in Mo as in other BCC metals. For the purposes of the present calculation, it is assumed that the operating slip planes are [110]. In this simple version, there are 12 possible cases, as shown in Table I.

The case for calculating the stress required to cause yielding in the longitudinal (extrusion) direction is simple. The tensile direction, $\hat{t}$, is $1 / \sqrt{2}$ [011], as indicated in Figure 4. Thus, the Schmid factors, $M$, are readily calculated for each slip system and these are also shown in Table $\mathrm{I} . \dagger$ When a shear stress, $\tau$, in one or more slip

$\dagger$ For simplicity, the descriptions for slip planes and directions include two directions. For example, the slip plane (110) in Table I includes both (110) and (110)

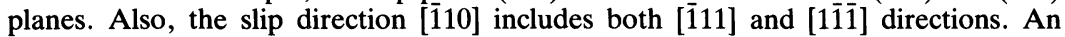
angle between the tensile direction and the direction normal to the slip plane, $\phi$, and an angle between the tensile direction and the slip direction, $\lambda$, range between 0 to $90^{\circ}$. That is, $0 \leq \cos \theta \leq 1$ and $0 \leq \cos \lambda \leq 1$. For calculations of $\cos \theta$ and $\cos \lambda$, in Table I, the slip planes and directions were chosen so that $\cos \theta \geq 0$ and $\cos \lambda \geq 0$, respectively. 


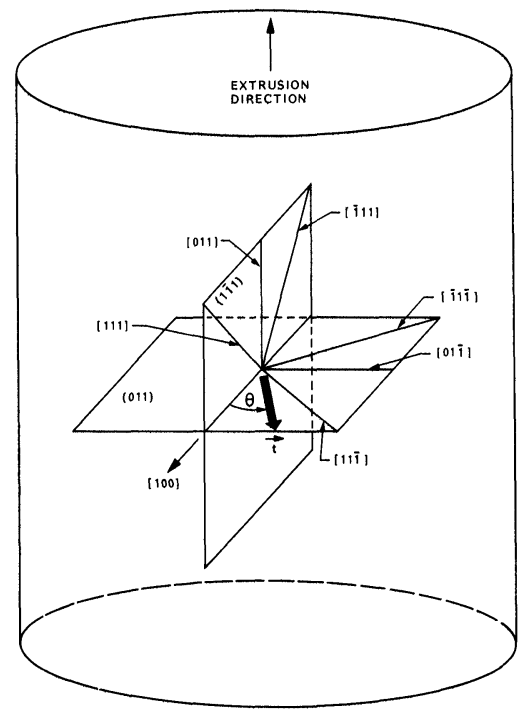

FIGURE 4 Available slip systems in a unit cell of a BCC metal bar containing a perfect (110) fiber texture.

TABLE I

Slip systems and Schmid factors for a direction parallel to the extrusion direction for BCC metals

\begin{tabular}{|c|c|c|c|c|}
\hline Slip Plune & Slip Direction & $\operatorname{Cos} \varphi$ & $\operatorname{Cos} \lambda$ & $M$ \\
\hline 110 & 111 & $1 / 2$ & $\sqrt{2 / 3}$ & $1 / \sqrt{6}$ \\
110 & $1 \overline{1} 1$ & $1 / 2$ & 0 & 0 \\
$1 \overline{1} 0$ & 111 & $1 / 2$ & $\sqrt{2 / 3}$ & $1 / \sqrt{6}$ \\
$1 \overline{1} 0$ & $11 \overline{1}$ & $1 / 2$ & 0 & 0 \\
011 & $1 \overline{1} 1$ & 1 & 0 & 0 \\
011 & $11 \overline{1}$ & 1 & 0 & 0 \\
$01 \overline{1}$ & 111 & 0 & $\sqrt{2 / 3}$ & 0 \\
$01 \overline{1}$ & 111 & 0 & $\sqrt{2 / 3}$ & 0 \\
101 & $11 \overline{1}$ & $1 / 2$ & 0 & 0 \\
101 & $i 11$ & $1 / 2$ & $\sqrt{2 / 3}$ & $1 / \sqrt{6}$ \\
$10 \overline{1}$ & 111 & $1 / 2$ & $\sqrt{2 / 3}$ & $1 / \sqrt{6}$ \\
$10 \overline{1}$ & 111 & $1 / 2$ & 0 & 0 \\
\hline
\end{tabular}


system reaches $\tau_{\text {crss }}$ (the critical resolved shear stress), the material is assumed to yield. In the case of the longitudinal direction, four slip systems yield at the same point and $\sigma_{y}=\tau_{\text {crss }} / M_{\max }=\sqrt{6} \tau_{\text {crss }}$.

The calculation of the stress required to cause yielding in the direction transverse to the extrusion direction is not so straightforward. In this case, the grains within an extruded bar all have [011] parallel to the extrusion direction, but can have random rotation along the extrusion direction or [011] direction. To calculate the $\sigma_{y}$ for this condition, a single-crystal system is assumed (Figure 4) and the tensile direction $(\vec{t})$ is rotated along the [011] direction. The yield stress is then calculated as a function of the rotation angle, $\theta$, that is measured from the [100] direction. This stress is averaged to estimate the $\sigma_{y}$ for the transverse direction.

Because of the symmetry inherent in the transverse cases, it is only required to calculate values for $\theta$ from 0 to $90^{\circ}$. Initially it is necessary to calculate $\vec{t}$ as a function of $\theta$. Since $\vec{t}=\left(t_{1}, t_{2}, t_{3}\right)$ and $t_{1}^{2}+t_{2}^{2}+t_{3}^{2}=1$, and since $\vec{t}$ is on the (011) plane, $t_{3}=-t_{2}$. Furthermore, $t_{1}=\cos \theta$, so that $t_{2}=-t_{3}=+(1 / \sqrt{2}) \sin \theta$. When $\theta=90^{\circ}, t_{2}=(1 / \sqrt{2})$ and $t_{3}=(-1 / \sqrt{2})$. Thus, because $\vec{t}=[\cos \theta$, $(1 / \sqrt{2}) \sin \theta,(-1 / \sqrt{2}) \sin \theta]$, the Schmid factors can be calculated for the 12 slip systems and these are shown in Table II. Five different functions for $M$ result and these can be rewritten as follows:

$$
\begin{gathered}
\frac{1}{\sqrt{6}}\left|\cos ^{2} \theta \pm \frac{1}{\sqrt{2}} \sin \theta \cos \theta\right|=\frac{1}{2 \sqrt{6}}\left|1+\cos 2 \theta \pm \frac{1}{\sqrt{2}} \sin 2 \theta\right| \\
\frac{1}{\sqrt{6}}\left|\cos ^{2} \theta-\sin ^{2} \theta \pm\left(\sqrt{2}-\frac{1}{\sqrt{2}}\right) \sin \theta \cos \theta\right| \\
=\frac{1}{2 \sqrt{6}}\left|2 \cos 2 \theta \pm\left(\sqrt{2}-\frac{1}{\sqrt{2}}\right) \sin 2 \theta\right| \\
\frac{1}{\sqrt{6}} 2 \sin \theta \cos \theta=\frac{1}{\sqrt{6}} \sin 2 \theta
\end{gathered}
$$

The values of Schmid factor, $M$, for each of these five functions are shown versus $\theta$ in Figure 5 .

The average value of stress, $\sigma_{\mathrm{av}}$, is then given as

$$
\sigma_{\mathrm{av}}=\frac{2}{\pi} \int_{0}^{\pi / 2} \sigma(\theta) \mathrm{d} \theta
$$


TABLE II

Slip systems and Schmid factors for a direction normal to the extrusion direction

\begin{tabular}{|c|c|c|c|c|}
\hline $\begin{array}{l}\text { Slip } \\
\text { Plane* }\end{array}$ & $\begin{array}{c}\text { Slip } \\
\text { Direction }\end{array}$ & $\operatorname{Cos} \varphi$ & $\operatorname{Cos} \lambda$ & M \\
\hline 110 & $\overline{111}$ & $\frac{1}{\sqrt{2}}\left(\cos \theta+\frac{1}{\sqrt{2}} \sin \theta\right)$ & $\frac{1}{\sqrt{3}}(\cos \theta)$ & $\frac{1}{\sqrt{6}}\left[\cos ^{2} \theta+\frac{1}{\sqrt{2}} \sin \theta \cos \theta\right]$ \\
\hline 110 & $1 \overline{1} 1$ & $\frac{1}{\sqrt{2}}\left(\cos \theta+\frac{1}{\sqrt{2}} \sin \theta\right)$ & $\frac{1}{\sqrt{3}}|\cos \theta-\sqrt{2} \sin \theta|$ & $\frac{1}{\sqrt{6}}\left|\cos ^{2} \theta-\sin ^{2} \theta-\left(\sqrt{2}-\frac{1}{\sqrt{2}}\right) \sin \theta \cos \theta\right|$ \\
\hline $1 \overline{1} 0$ & 111 & $\frac{1}{\sqrt{2}}\left|\cos \theta-\frac{1}{\sqrt{2}} \sin \theta\right|$ & $\frac{1}{\sqrt{3}} \cos \theta$ & $\frac{1}{\sqrt{6}}\left|\cos ^{2} \theta-\frac{1}{\sqrt{2}} \sin \theta \cos \theta\right|$ \\
\hline $1 \overline{1} 0$ & $11 \overline{1}$ & $\frac{1}{\sqrt{2}}\left|\cos \theta-\frac{1}{\sqrt{2}} \sin \theta\right| \mid$ & $\frac{1}{\sqrt{3}}(\cos \theta+\sqrt{2} \sin \theta)$ & $\frac{1}{\sqrt{6}} \mid \cos ^{2} \theta-\sin ^{2} \theta+\left(\sqrt{2}-\frac{1}{\sqrt{2}}|\sin \theta \cos \theta|\right.$ \\
\hline $01 \overline{1}$ & 111 & $\frac{1}{\sqrt{2}}(2 \sin \theta)$ & $\frac{1}{\sqrt{3}} \cos \theta$ & $\frac{1}{\sqrt{6}}(2 \sin \theta \cos \theta)$ \\
\hline $01 \overline{1}$ & $\tilde{1} 11$ & $\frac{1}{\sqrt{2}}(2 \sin \theta)$ & $\frac{1}{\sqrt{3}}(\cos \theta)$ & $\frac{1}{\sqrt{6}}(2 \sin \theta \cos \theta)$ \\
\hline 101 & $11 \tilde{1}$ & $\frac{1}{\sqrt{2}}\left|\cos \theta-\frac{1}{\sqrt{2}} \sin \theta\right|$ & $\frac{1}{\sqrt{3}}(\cos \theta+\sqrt{2} \sin \theta)$ & $\frac{1}{\sqrt{6}}\left|\cos ^{2} \theta-\sin ^{2} \theta+\left(\sqrt{2}-\frac{1}{\sqrt{2}}\right) \sin \theta \cos \theta\right|$ \\
\hline 101 & $\overline{1} 11$ & $\frac{1}{\sqrt{2}}\left|\cos \theta-\frac{1}{\sqrt{2}} \sin \theta\right|$ & $\frac{1}{\sqrt{3}}(\cos \theta)$ & $\frac{1}{\sqrt{6}}\left|\cos ^{2} \theta-\frac{1}{\sqrt{2}} \sin \theta \cos \theta\right|$ \\
\hline $10 \overline{1}$ & 111 & $\frac{1}{\sqrt{2}}\left(\cos \theta+\frac{1}{\sqrt{2}} \sin \theta\right)$ & $\frac{1}{\sqrt{3}} \cos \theta$ & $\frac{1}{\sqrt{6}}\left[\cos ^{2} \theta+\frac{1}{\sqrt{2}} \sin \theta \cos \theta\right]$ \\
\hline $10 \overline{1}$ & $1 \overline{1} 1$ & $\frac{1}{\sqrt{2}}\left(\cos \theta+\frac{1}{\sqrt{2}} \sin \theta\right)$ & $\frac{1}{\sqrt{3}}|\cos \theta-\sqrt{2} \sin \theta|$ & $\frac{1}{\sqrt{6}}\left|\cos ^{2} \theta-\sin ^{2} \theta-\left(\sqrt{2}-\frac{1}{\sqrt{2}}\right) \sin \theta \cos \theta\right|$ \\
\hline
\end{tabular}

${ }^{*}$ For the (011) plane, $\cos \theta=0$

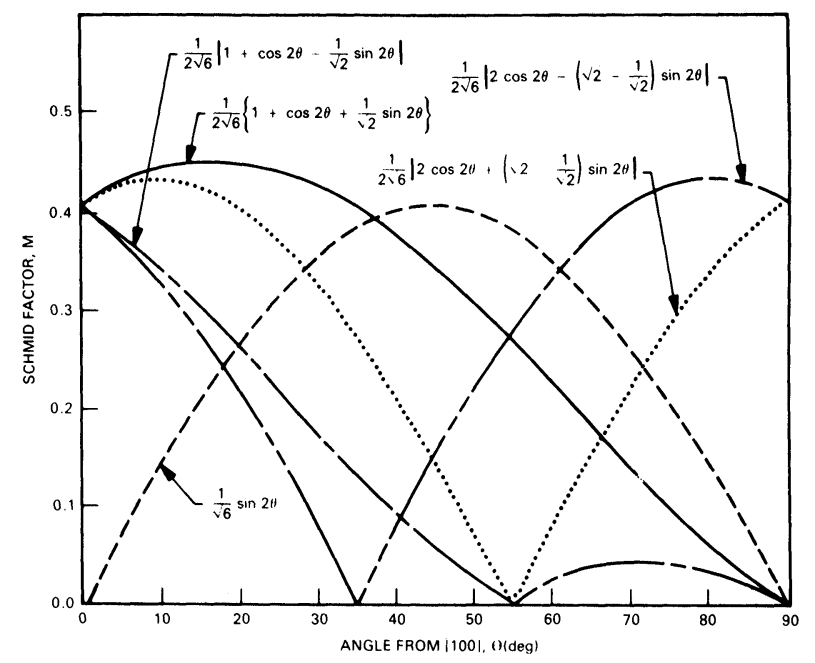

FIGURE 5 Schmid Factor, $M$, as a function of $\theta$ for the five possible cases. 
This can be expressed in terms of the critical resolved shear stress, $\tau_{\text {crss }}$, and the Schmid factor as

$$
\sigma_{\mathrm{av}}=\frac{2 \tau_{\mathrm{crss}}}{\pi} \int_{0}^{\pi / 2} \frac{\mathrm{d} \theta}{M_{\max }(\theta)}
$$

or in final form

$$
\frac{1}{M_{\mathrm{av}}}=\frac{2}{\pi} \int_{0}^{\pi / 2} \frac{\mathrm{d} \theta}{M_{\max }(\theta)}
$$

This function was computed numerically and shown to be equal to 2.41. Thus:

$$
\sigma_{\mathrm{av}}=2.413 \tau_{\mathrm{crss}}
$$

It may thus be concluded that in a bar containing a perfect [110] fiber texture, the difference between the anticipated yield strengths for the longitudinal direction $\left(\sigma=\sqrt{6} \tau_{\text {crss }}=2.45 \tau_{\text {crss }}\right)$ and the transverse direction $\left(\sigma=2.41 \tau_{\text {crss }}\right)$ is extremely small.

An experimental study was performed to determine the validity of this prediction. Six samples of both longitudinal and transverse configurations were used for TZM and unalloyed Mo. The yield strengths are as follows (the numbers in parenthesis refer to the standard deviations):

$\begin{array}{ccc} & \begin{array}{c}\text { Longitudinal } \\ \text { (MPa) }\end{array} & \begin{array}{c}\text { Transverse } \\ \text { (MPa) }\end{array} \\ \text { TZM } & 636(24) & 644(21) \\ \text { Mo } & 697(16) & 730(25)\end{array}$

As many be seen, in agreement with prediction, the experimentally determined yield strengths are essentially the same in the longitudinal and transverse direction (within error) for both Mo and TZM. Thus, it is concluded that differences in yield strength between the longitudinal and transverse directions of a Mo or TZM bar containing a [110] texture are not a factor in promoting the brittle behaviour of transversely oriented samples.

\section{Acknowledgements}

The authors would like to thank Mr. W. C. Coons for the optical photomicrographs. This work was funded by the Lockheed Independent Research and Development Program. 


\section{References}

Hosford, W. F. (1964) Trans. TMS-AIME, 230, 12-15.

Peck, J. F., and Thomas, D. A. (1961). Trans. TMS-AIME, 221, 1240-1247.

Tietz, T. E., and Perkins, R. A. (1964). Jour. Spacecraft and Rockets, 1, 225-234.

Wadsworth, J., Packer, C. M., Chewey, P. M., and Coons, W. C. (1984a). Metall. Trans., Ser. A, 15A, 1741-1752.

Wadsworth, J., Packer, C. M., and Coons, W. C. (1984b). Proc. Symp. Phys. Metall. Technol. Molybdenum and Its Alloys, AMAX Speciality Metals Corp., Ann Arbor, MI, p13-19. 